Antimatter and Gravity (WAG 2013)

International Journal of Modern Physics: Conference Series

Vol. 30 (2014) 1402006 (4 pages)

(C) The Authors

DOI: $10.1142 / \mathrm{S} 2010194514020066$

\title{
Foreword
}

The 2nd International Workshop on Antimatter and Gravity (WAG 2013) took place on 13-15 November 2013 at the University of Bern, Switzerland, hosted by the Albert Einstein Center for Fundamental Physics (AEC),following the first workshop held in 2011 at the Institut Henri Poincaré in Paris. The members of the International Advisory Committee and of the Local Organizing Committee are listed below. Details can be found on the conference web site.*

The purpose of this meeting was to review the experimental and theoretical aspects of the interaction of antimatter with gravity. Tests of the weak equivalence principle (WEP) with matter on ground and in space where reviewed, and the ensuing constraints on the gravitational interaction between matter and antimatter were lively discussed. New ideas to measure the gravitational free-fall of antimatter with, e.g. positronium, muonium and antihydrogen were presented. Progress reports on the antimatter experiments at the CERN Antiproton Decelerator and atthe future facilities ELENA (CERN) and FLAIR (GSI) were delivered. A session was also devoted to the relevance of antimatter with respect to Dark Energy and Dark Matter in the Universe.

Appropriately, the WEP being a cornerstone of Einstein's General Relativity, a visit of the house at the Kramgasse 49, where Einstein spent the year 1903 until his "Annus Mirabilis" in 1905, was organized. A social dinner at the "Altes Tramdepot" was part of the social programme.

The Workshop (28 plenary invited talks) took place in the "Kuppelraum" of the main building of the University, and was attended by 69 registered participants plus additional guests.

The articles published in these Proceedings were peer-reviewed. Nevertheless, the scientific accuracy and statements made in the papers rest with the sole responsibilities of the authors.

The conference was sponsored by the Laboratory of High Energy Physics of the AEC. We wish to thank Ms. I. Neeser and Ms. U. Witschi for assisting us with the organization of the event, such as the registration of participants and the coffee breaks.

Claude Amsler \& Paola Scampoli

*https://indico.cern.ch/event/227924/ 


\section{International Advisory Committee}

M. Blau (University of Bern, Switzerland)

G. Chardin (CNRS/IN2P3, France)

M. Doser (CERN, Switzerland)

T. Jolicoeur (CNRS/Université Paris-Sud, France)

A. Kostelecky (Indiana University, USA)

C. Lämmerzahl (University of Bremen, Germany)

M. Oberthaler (Heidelberg University, Germany)

W. Oelert (Mainz University, Germany)

V.V. Nesvizhevsky (ILL, Grenoble, France)

P. Perez (CEA, France)

Y. Sacquin (CEA, France)

G. Testera (INFN Genova, Italy)

P. von Ballmoos (IRAP, Toulouse, France)

C. Will (University of Florida, USA)

Y. Yamazaki (RIKEN, Japan)

\section{Local Organizing Committee}

Claude Amsler (Chair)

Akitaka Ariga

Antonio Ereditato

Ciro Pistillo

Paola Scampoli

James Storey

Marcella Esposito (Conference Secretary) 


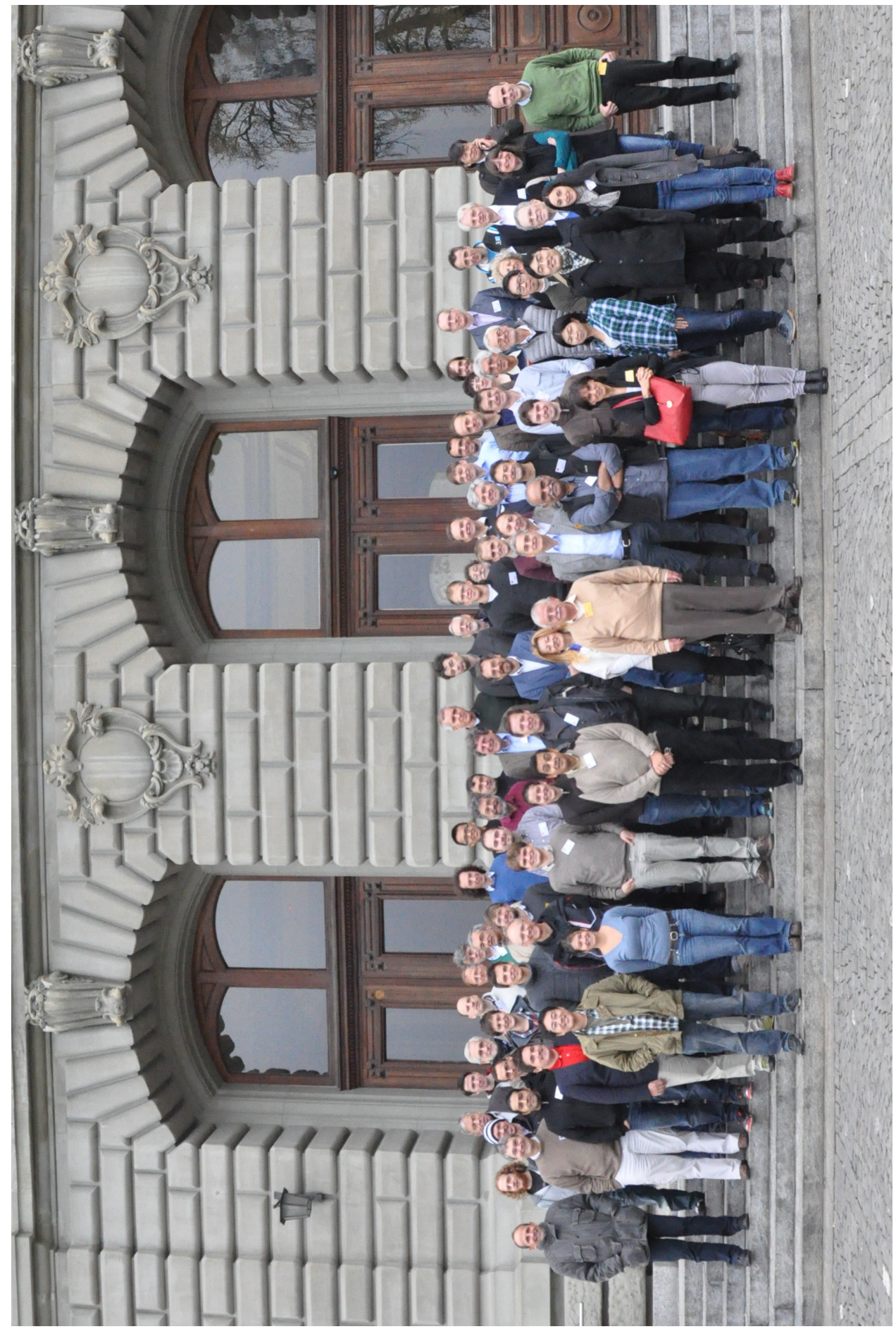




\section{Participants}

AMSLER, Claude ARIGA, Akitaka ARIGA, Tomoko* BAUMANN, Henri* BENOIT-LÉVY, Aurélien* BLANCHET, Luc* BORDÉ, Christian* BRÄUNIG, Philippe* CABBOLET, Marcoen CARAVITA Ruggero CASSIDY, David* CHARLTON, Michael* CHLOÉ, Malbrunot CARLI, Christian* CRIVELLI, Paolo* DE MIRANDA SILVEIRA, Daniel DICKERSON, Susannah* DUFOUR, Gabriel*

EREDITATO, Antonio

FAJANS, Joel*

FUTAMASE, Toshifumi GERBER, Paul GLIGOROVA, Angela HAAS, Samuel HAIDER Stefan HASHIMOTO, Koji* HASLINGER, Philipp HILICO, Laurent*

HOLZSCHEITER, Michael* $^{*}$ JANKOWIAK, Martin* JOLICOEUR, Thierry KARSHENBOIM, Savely* KAWADA, Jiro
AEC/LHEP, Bern University, Bern AEC/LHEP, Bern University, Bern AEC/LHEP, Bern University, Bern Federal Institute of Metrology METAS, Bern University College, London Institut d'Astrophysique de Paris Académie des Sciences, Paris Kirchhoff-Institute for Physics, Heidelberg Vrije Universiteit, Brussel INFN, Genoa University College, London Swansea University, Swansea CERN, Geneva CERN, Geneva Institute for Particle Physics, ETH, Zurich Federal University of Rio de Janeiro, Rio de Janeiro

Stanford University, Stanford

Laboratoire Kastler-Brossel, Université Pierre et Marie Curie, Paris AEC/LHEP, Bern University, Bern U.C. Berkeley, Berkeley Astronomical Institute, Tohoku Universit, Sendai Gerber Molecular Design, Amden University of Bergen, Bergen

ETH, Zurich CERN, Geneva

Osaka University, RIKEN

University of Vienna VCQ, Vienna

Laboratoire Kastler Brossel, Université d'Evry Val d'Essone, Evry

University of New Mexico, Albuquerque

Stanford University, Stanford

CNRS and University Paris-XI, Orsay Mpq \& Amp; Pulkovo OBS, Garching AEC/LHEP, Bern University, Bern 
KHAW, Kim Siang

KIMURA, Mitsuhiro

KIRCH, Klaus*

KRASNICKY, Daniel*

LAMMERZAHL, Claus

LEBRUN, Patrice

LEHNER, Sebastian

LISZKAY, Laszlo

MAURY, Stephan

NOBILI, Anna Maria*

OBERTHALER, Markus

OELERT, Walter

PEREZ, Patrice

PHILLIPS, Thomas

PISTILLO, Ciro

PORTER, Sarah

PROTASOV, Konstantin

QUINT, Wolfgang*

SACQUIN, Yves

SALATI, Pierre*

SCAMPOLI, Paola

SIMON, Martin

SKINNER, Gerry*

STOREY, James

SUBIETA VASQUEZ,

Martin Alfonso

TASSON, Jay*

TESTERA, Gemma

TRANQUILLE, Gerard

UNNIKRISHNAN, C.S.*

VAN DER WERF, Dirk*

VON BALLMOOS, Peter

VORONIN, Alexei*

WIDMANN, Eberhard

WOLF, Sebastian

WURTELE, Jonathan

ZAVATARELLI, Sandra
ETH, Zurich

AEC/LHEP, Bern University, Bern

PSI \& ETH, Zurich

INFN, Genoa

Center of Applied Space Technology and

Microgravity, Bremen

IPNL, Lyon

Stefan Meyer Institut, Vienna

IRFU CEA Saclay, Gif-Sur-Yvette

CERN, Geneva

Dipartimento di Fisica "E. Fermi", University of Pisa, Pisa

Kirchhoff-Institut for Physics, Heidelberg

Johannes Gutenberg-University, Mainz

CEA Saclay, Gif-Sur-Yvette

Duke University, Durham

AEC/LHEP, Bern University, Bern

CHUV, Lausanne

Laboratory for Subatomic Physics and Cosmology,

Grenoble

Helmholtzzentrum GSI, Darmstadt

Laboratoire Kastler Brossel, Paris

LAPTH, Annecy

Dipartimento di Fisica, Università di Napoli

Federico II, Napoli

Stefan Meyer Institut, Vienna

Keystone College, La Plume, Pennsylvania

AEC/LHEP, Bern University, Bern

University of Brescia, Brescia

Carleton College, Northfield, Minnesota

INFN, Genoa

CERN,Geneva

Tata Institute of Fundamental Research, Mumbai

Swansea University, Swansea

Institut de Recherche en Astrophysique et

Planétologie, Toulouse

P.N. Lebedev Physical Institute, Moscow

Stefan Meyer Institut, Vienna

Johannes Gutenberg University, Mainz

University of California, Berkeley

INFN, Genoa 